\title{
Effectiveness of Hammock Positioning in Reducing Pain and Improving Sleep-Wakefulness State in Preterm Infants
}

\author{
Camila Gemin Ribas, Marimar Gorette Andreazza, Valéria Cabral Neves, and Silvia Valderramas
}

BACKGROUND: Hammock positioning is now frequently used with preterm infants admitted to ICUs. However, few studies have investigated the extent to which hammock positioning reduces pain and improves the sleep-wakefulness state compared with traditional positioning. METHODS: Twenty-six clinically stable newborns with gestational ages from 30 to 37 weeks who were breathing spontaneously were randomly assigned to 2 groups: a hammock-positioning group $(n=13)$, in which newborns were placed in hammocks in the lateral position, and a traditional-positioning group $(n=13)$, in which they were kept nested, also in the lateral position. The following variables were evaluated at the beginning and the end of the treatment: pain (with the Premature Infant Pain Profile, and Neonatal Facial Coding System), sleep-wakefulness state (with the Brazelton Neonatal Behavioral Assessment Scale), heart rate, breathing frequency, and peripheral $\mathrm{S}_{\mathrm{pO}_{2}}$. RESULTS: The subjects in the hammock-positioning group showed an improvement in pain compared with the traditional-positioning group(Premature Infant Pain Profile score, $2.62 \pm 1.89$ vs $2.31 \pm 1.97$, $\Delta P=.008)$ and sleep-wakefulness state score $(2.08 \pm 0.64$ vs $1.23 \pm 0.44, \Delta P<.001)$, reduced heart rate $(151.69 \pm 5.44$ vs $142.77 \pm 5.18$ beats $/ \mathrm{min}, \Delta P<.001)$, breathing frequency $(52.31 \pm 4.05 \mathrm{vs}$ $50.23 \pm 2.55$ beats $/ \mathrm{min}, \Delta P=.024)$, and increased peripheral $\mathrm{S}_{\mathrm{pO}_{2}}(94.69 \pm 2.14 \mathrm{vs} 98.00 \pm 1.22 \%$, $\Delta P<.001)$. CONCLUSIONS: Hammock positioning was an effective treatment option to reduce pain and improve sleep-wakefulness state. It also helped to reduce heart rate and breathing frequency, and to increase peripheral $\mathrm{S}_{\mathrm{pO}_{2}}$, which made it a treatment option for preterm infants. Key words: preterm infant; physical therapy specialty; patient positioning; respiratory care; respiratory therapy. [Respir Care 2019;64(4):384-389. () 2019 Daedalus Enterprises]

\section{Introduction}

Premature birth is a consequence of a variety of unpredictable circumstances. An infant is considered premature if born with a gestational age of $<37$ weeks and extremely

\footnotetext{
Ms Ribas, Ms Andreazza, and Ms Neves are affiliated with the Complexo Hospital de Clínicas, Federal University of Paraná, Curitiba, Brazil. Dr Valderramas is affiliated with the Department of Prevention and Rehabilitation in Physical Therapy, and Internal Medicine Masters/Doctorate Programme, Federal University of Paraná, Curitiba, Brazil.
}

The authors have disclosed no conflicts of interest.

Correspondence: Silvia Valderramas PT PhD, Department of Prevention and Rehabilitation in Physical Therapy, Federal University of Paraná, Av. Cel. Heráclito dos Santos, CP 19031, Curitiba, Paraná, CEP 81531900, Brazil. E-mail: svalderramas@uol.com.br.

DOI: $10.4187 /$ respcare. 06265 premature when the gestational age at birth is $<28$ weeks. $^{1}$ Preterm infants are now managing to survive the challenge of premature birth at ever-younger gestational ages, and survival rates of extremely preterm infants as high as $80 \%$ have been reported. This can be attributed to advances in prenatal care and technologic improvements in neonatal ICUs $^{2}$ During their long hospital stay, this population is routinely exposed to a stressful environment and painful interventions, ${ }^{3}$ which can trigger clinical instability in these individuals. Non-pharmacologic interventions that alleviate pain and make it easier for the infant to sleep, such as positioning or tactile stimuli, therefore, are necessary in addition to pharmacologic interventions. ${ }^{4,6}$

The positioning of preterm infants in neonatal ICUs can have a positive effect on ventilation and lung perfusion parameters, as shown in a study by Paschoalotto et al, ${ }^{7}$ in which newborns were placed nested in the lateral position, also known as traditional positioning. However, few studies have described the clinical effects of hammock 
positioning on preterm infants, and the results are still controversial because of the limitations of the assessment methods used. ${ }^{8-10}$ In hammock positioning, the newborn is placed on a rectangle of cloth (hammock) that is attached to the incubator at its extremities, which simulates the intrauterine position. This positioning stimulates the development of flexion, promotes symmetry, and, therefore, is less harmful in terms of motor behavior. It is a simple, inexpensive, noninvasive intervention, and alternative position for infants who need to stay in the neonatal ICU for a prolonged period. ${ }^{8}$

\section{See the Related Editorial on Page 489}

In this clinical trial, we hypothesized that the use of hammock positioning would lead to clinical and physiologic improvements in preterm infants compared with traditional positioning. The aim of the study was to assess the effectiveness of hammock positioning in reducing pain and improving the sleep-wakefulness state, heart rate, breathing frequency, and peripheral $\mathrm{S}_{\mathrm{pO}_{2}}$ in preterm infants compared with traditional positioning.

\section{Methods}

The study was a randomized controlled clinical trial carried out in the neonatal ICU at the Hospital de Clínicas Complex, Federal University of Paraná, Curitiba, Parana, Brazil, from March to June 2015. It followed the Consolidated Standards of Reporting Trials recommendations and was approved by the institutional ethics committee (878051/ 2015) and registered at the Brazilian Clinical Trials Registry (RBR-8S8C8C). The infants' parents or guardians signed a voluntary informed-consent form that explained all the study aims, procedures, possible risks, and benefits.

The inclusion criteria were the following: having a gestational age of between 30 and 37 weeks, breathing spontaneously, and being clinically stable. Exclusion criteria were the following: having a gestational age of $<30$ weeks or $>37$ weeks presenting with hemodynamic instability, being on mechanical ventilation, or presenting with congenital facial anomalies that prevented assessment of pain. The groups were characterized in terms of gestational age, weight, and sex. Pain was assessed with the Premature Infant Pain Profile ${ }^{11,12}$ and the Neonatal Facial Coding System $^{12}$ scales, and the sleep-wakefulness state was assessed with the Brazelton Neonatal Behavioral Assessment Scale. ${ }^{13}$ The clinical variables heart rate, breathing frequency, and $\mathrm{S}_{\mathrm{pO}_{2}}$ were also measured. All assessments were performed by a single assessor $(\mathrm{V} \mathrm{C} \mathrm{N})$ who was blinded to the interventions used in each group.

After they had been assessed, the subjects were randomly assigned to 2 groups: the hammock-positioning

\section{QUICK LOOK}

\section{Current knowledge}

Hammock position simulates the intrauterine position; newborns are placed on a rectangular piece of cloth attached at the ends to the incubator. It is a simple, inexpensive alternative position for preterm infants admitted to the ICU.

\section{What this article contributes to our knowledge}

Hammock placement was an effective treatment option for reducing pain and improving sleep-wakefulness state and can be used as an option in the non-pharmacologic treatment of preterm infants.

group, in which they were placed in hammocks in the lateral position and the traditional-positioning group, in which they were kept nested in the lateral position. The interventions (hammock or traditional positioning) were performed for $2 \mathrm{~h}$ every day for 5 consecutive days and were supervised by 2 previously trained physical therapists ( $\mathrm{C} \mathrm{GR}$ and M G A). A period of $2 \mathrm{~h}$ was chosen based on the duration of the sleep-wakefulness cycle ${ }^{13}$ and the infant's nap period (when no interventions were performed) in the neonatal ICU. Outcomes were assessed $10 \mathrm{~min}$ before and immediately after the 2 -h intervention for 5 consecutive days.

\section{Interventions}

The subjects allocated to the hammock-positioning group were placed in the lateral position in hammocks made of swaddling cloth, which measured $30 \mathrm{~cm}$ long $\times 25 \mathrm{~cm}$ wide. The hammocks were attached to the incubators by straps at both ends (Figure 1). Interventions were performed in the neonatal ICU and were immediately interrupted if any hemodynamic complications occurred or respiratory effort was observed.

\section{Primary Outcome}

The primary outcome was pain, which was assessed with the Premature Infant Pain Profile and the Neonatal Facial Coding System scales. Each scale requires detailed knowledge of the instrument. The Premature Infant Pain Profile uses gestational age, alertness, heart rate, $\mathrm{S}_{\mathrm{pO}_{2}}$, and facial expression (furrowed brow, eye squeeze, and nasolabial furrow). ${ }^{11,12}$ The Neonatal Facial Coding System analyzes the following facial expressions frequently associated with pain: brow bulge, eye squeeze, deepening of the nasolabial furrow, open lips, mouth stretch (hor- 


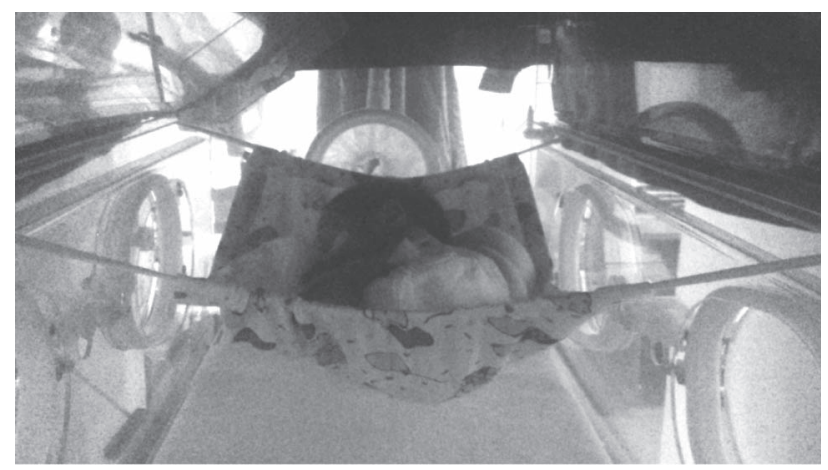

Fig. 1. A preterm infant positioned in a hammock.

izontal or vertical), tongue tautening, tongue protrusion, and chin quiver. One point (of a maximum of 8) is assigned for each facial expression. Pain is considered present when $\geq 3$ of these facial movements are observed during the evaluation. ${ }^{12}$ The Neonatal Facial Coding System scale is the most widely applied scale in clinical practice because of its ease of use.

\section{Secondary Outcomes}

The secondary outcomes were the sleep-wakefulness state and the physiologic parameters heart rate, breathing frequency, and $\mathrm{S}_{\mathrm{pO}_{2}}$. The sleep-wakefulness state was assessed with the Brazelton Behavioral Assessment Scale, which ranges from 1 to 6 , which corresponds to the following states: deep sleep, light sleep, drowsy, alert, eyes open, and crying, respectively. ${ }^{13}$ Heart rate and $\mathrm{S}_{\mathrm{pO}_{2}}$ were measured with a Datex Ohmeda 3800 pulse oximeter. Bradycardia and tachycardia were considered to correspond to a heart rate of $<80$ beats $/ \mathrm{min}$ and $>160$ beats $/ \mathrm{min}$, respectively. Hypoxia was considered present when $\mathrm{S}_{\mathrm{pO}_{2}}$ was $<90 \% .{ }^{14}$ Breathing frequency was measured with a Vollo 500-memory stopwatch. Bradypnea and tachypnea were considered to correspond to a frequency of $<40$ breaths/min and $>60$ breaths/min, respectively.

\section{Sampling and Randomization}

Sampling was non-probabilistic, and all the patients in the neonatal ICU in the study period (3 months) who met the inclusion criteria were included in the study. When considering the resources available for data collection, the rate of admission to the neonatal ICU, the small sample size in previous studies of this kind ${ }^{8}$ and a post hoc calculation of the power of the study by using an effect size of 1.13 based on the Cohen d, the sample size was fixed at 26 preterm infants. The participants were randomly assigned to the groups by using a simple randomization procedure (computer-generated random numbers). This pro-

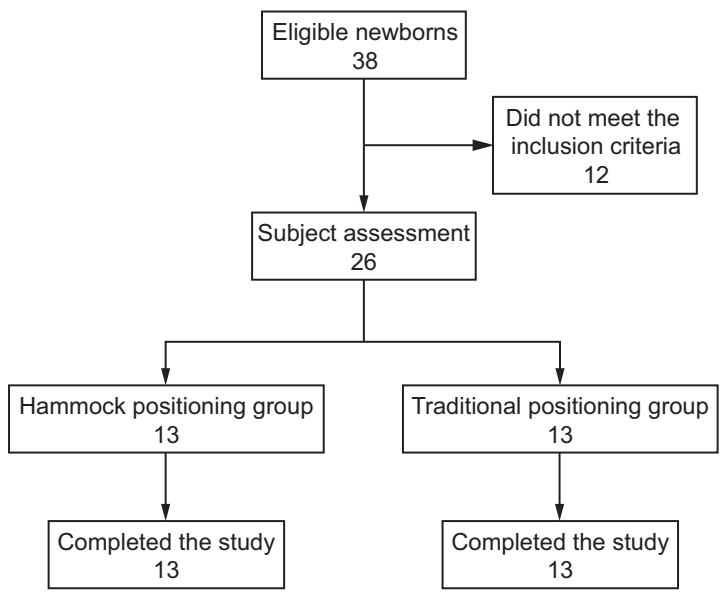

Fig. 2. Flow chart. Assessment included the Premature Infant Pain Profile, Neonatal Facial Coding System, and the Brazelton Neonatal Behavioral Assessment scales as well as physiologic variables.

cedure was carried out by an independent physical therapist (SV).

\section{Statistical Analysis}

The data were analyzed with SPSS version 16.0 for Windows (SPSS, Chicago, IL). The Shapiro-Wilks and the Levene tests were used to test for normality and homogeneity of the variance, respectively. The results were presented as mean \pm SD and median and 25-75 percentiles. Differences between the groups were analyzed with the Mann-Whitney test and Student $t$ test according to the type of variable and the distribution of the data. The chi-square test was used for categorical variables. A significance level of $P<.05$ was used. Post hoc analysis of the statistical power was performed with $\mathrm{G}^{*}$ Power 3.1.15

\section{Results}

Thirty-eight preterm infants were recruited for the study. Of these, 26 met the inclusion criteria and were each randomly assigned to a group (hammock-positioning group, $n=13$; traditional-positioning group, $n=13$ ) (Figure 2). All the participants assigned to the groups completed the study. There were no statistically significant differences in baseline characteristics between the 2 groups (Table 1).

\section{Pain and Sleep-Wakefulness State}

The preterm infants allocated to the hammock-positioning group had significantly less pain (Premature Infant Pain Profile) and a better sleep-wakefulness state (Brazelton Neonatal Behavioral Assessment Scale) than the subjects allo- 
cated to the traditional-positioning group (Table 2). Statistically significant differences were also observed for heart rate, breathing frequency, and $\mathrm{S}_{\mathrm{pO}_{2}}$, with better post-intervention results in the hammock-positioning group (Table 3).

\section{Discussion}

At the end of the treatment, the hammock-positioning group had less pain and improved sleep-wakefulness state, heart rate, breathing frequency, and $\mathrm{S}_{\mathrm{pO}_{2}}$ compared with those in the traditional-positioning group. The baseline characteristics of the preterm infants in the study, which included low gestational age, did not influence the results when the population sample was divided into the 2 intervention groups. Preterm infants have all the functional and neurochemical components needed to receive and transmit pain signals. ${ }^{16}$ However, their responses to stimuli are non-specific and disorganized

Table 1. Baseline Characteristics of the Subjects

\begin{tabular}{lccc}
\hline \hline \multicolumn{1}{c}{ Variable } & $\begin{array}{c}\text { Traditional- } \\
\text { Positioning } \\
\text { Group } \\
(n=13)\end{array}$ & $\begin{array}{c}\text { Hammock- } \\
\text { Positioning } \\
\text { Group } \\
(n=13)\end{array}$ & $P$ \\
\hline Female/male, $n$ & $10 / 3$ & $8 / 5$ & .67 \\
Corrected age, mean \pm SD mo & $33.1 \pm 1.0$ & $32.31 \pm 1.70$ & .18 \\
Apgar score, mean \pm SD & $8.00 \pm 0.82$ & $7.85 \pm 0.80$ & .63 \\
Weight, mean \pm SD kg & $1.31 \pm 0.48$ & $1.62 \pm 0.51$ & .13 \\
\hline
\end{tabular}

because myelinization of the nervous system is incomplete and the endogenous pain control systems that modulate pain are still immature. The pain experienced by preterm infants, therefore, is much greater and more acute than that felt by older children or adults, which leads to physical and psychological discomfort and suffering. ${ }^{16}$

Exposure to pain is one of the most harmful factors associated with the extrauterine environment and can affect brain development and respiratory, cardiovascular and metabolic stability, which increases neonatal morbidity and mortality rates. ${ }^{17}$ Pain-relief procedures increase homeostasis and stability in preterm infants and are essential in the care of these infants to enable them to survive the stress of a neonatal ICU. ${ }^{18}$ The results of the present study showed that the use of a simple non-pharmacologic method, such as hammock positioning, alleviated pain in $11.83 \%$ by the Premature Infant Pain Profile. To the best of our knowledge, this was the first randomized clinical trial to show the effectiveness of hammock positioning in reducing pain in preterm infants.

The development of sleep-wakefulness cycles starts during fetal life and continues into the postnatal period, with close links to maturation of the central nervous system. In preterm infants, sleep-wakefulness cycles have a polyphasic pattern, which alternates every 3 to 4 h. ${ }^{19}$ Rapid eye movement and non-rapid eye movement cycles are considered essential for neurodevelopment, learning, memory, and preservation of brain plasticity throughout the indi-

Table 2. Differences in Pain Variables (Premature Infant Pain Profile and Neonatal Facial Coding System) and Sleep-Wakefulness State (Neonatal Behavioral Assessment Scale) Between the Groups

\begin{tabular}{|c|c|c|c|c|c|}
\hline \multirow{2}{*}{ Variable } & \multicolumn{2}{|c|}{$\begin{array}{l}\text { Traditional-Positioning } \\
\quad \text { Group }(n=13)\end{array}$} & \multicolumn{2}{|c|}{$\begin{array}{l}\text { Hammock-Positioning } \\
\text { Group }(n=13)\end{array}$} & \multirow{2}{*}{$\Delta P^{*}$} \\
\hline & Pre & Post & Pre & Post & \\
\hline Premature Infant Pain Profile & $5.77 \pm 1.54$ & $2.62 \pm 1.89$ & $7.00 \pm 2.04$ & $2.31 \pm 1.97$ & .008 \\
\hline Neonatal Facial Coding System & $3.69 \pm 1.75$ & $1.92 \pm 1.26$ & $3.92 \pm 0.64$ & $1.15 \pm 1.34$ & .10 \\
\hline Neonatal Behavioral Assessment Scale & $4.00 \pm 0.91$ & $2.08 \pm 0.64$ & $4.15 \pm 0.90$ & $1.23 \pm 0.44$ & $<.001$ \\
\hline
\end{tabular}

Table 3. Differences in Physiologic Variables Between the Groups

\begin{tabular}{|c|c|c|c|c|c|}
\hline \multirow{2}{*}{ Variable } & \multicolumn{2}{|c|}{$\begin{array}{l}\text { Traditional-Positioning } \\
\quad \text { Group }(n=13)\end{array}$} & \multicolumn{2}{|c|}{$\begin{array}{l}\text { Hammock-Positioning } \\
\quad \text { Group }(n=13)\end{array}$} & \multirow{2}{*}{$P^{*}$} \\
\hline & Pre & Post & Pre & Post & \\
\hline Heart rate, beats/min & $152.46 \pm 6.25$ & $151.69 \pm 5.44$ & $152.00 \pm 8.19$ & $142.77 \pm 5.18$ & $<.001$ \\
\hline Breathing frequency, breaths/min & $52.62 \pm 3.86$ & $52.31 \pm 4.05$ & $55.38 \pm 8.64$ & $50.23 \pm 2.55$ & .02 \\
\hline Peripheral $\mathrm{S}_{\mathrm{pO}_{2}}, \%$ & $93.23 \pm 1.79$ & $94.69 \pm 2.14$ & $93.00 \pm 1.91$ & $98.00 \pm 1.22$ & $<.001$ \\
\hline
\end{tabular}


vidual's life. ${ }^{14,20}$ In view of this and when considering the environment in a neonatal ICU, therapeutic measures should be implemented to facilitate sleep cycles ${ }^{20}$ and ensure the comfort of the preterm infant. ${ }^{14}$

Our results showed that preterm infants placed in hammocks had an improvement of $41 \%$ in the sleep-wakefulness state. In addition, after $2 \mathrm{~h}, 10$ of the preterm infants $(77 \%)$ in the hammock positioning group were in deep sleep compared with $2(16 \%)$ in the traditional positioning group. Keller et $\mathrm{al}^{9}$ indicate that hammock positioning can help preterm infants overcome the effects of extrauterine forces, the absence of containment, and other sensory influences that affect the development of the immature brain. They found that preterm infants placed in hammocks for $3 \mathrm{~h}$ per day over $10 \mathrm{~d}$ had greater neuromuscular maturity than preterm infants kept in the prone position.

Peaceful sleep is known to be associated with increased lung volume, better organization of sleep and wakefulness states, physiologic stability and, consequently, weight gain. ${ }^{21}$ Sleep deprivation in preterm infants in neonatal ICUs may worsen the clinical status of these infants and delay discharge from hospital. ${ }^{22}$ The observations described above corroborate the results of the present study, in which the preterm infants placed in hammocks had an improvement of 6,4 , and $3.5 \%$ in heart rate, breathing frequency, and $\mathrm{S}_{\mathrm{pO}_{2}}$, respectively.

Changes in physiologic variables are known to be dependent on body position. ${ }^{21}$ Ideal positioning, therefore, is fundamental to mitigate the consequences of the mechanical limitations of the preterm infant's respiratory system. ${ }^{8}$ Costa et $\mathrm{al}^{8}$ studied heart rate in preterm infants, divided into 2 groups: hammock positioning $(n=30)$ and nest positioning $(n=30)$ (each participant was his or her own control, ie, each infant received the test intervention and, at a different time, the control intervention). A statistically significant, yet safe, reduction in heart rate was observed in the hammock-positioning group, which corroborated our findings. In contrast, Zanardo et al, ${ }^{23}$ who also used hammock positioning as an intervention in 15 preterm infants, failed to find statistically significant changes in heart rate and $\mathrm{S}_{\mathrm{pO}_{2}}$. This contrasts with the present study, in which the subjects in the hammock-positioning group had reduced heart rate and increased $\mathrm{S}_{\mathrm{pO}}$. Jesus et al ${ }^{10}$ observed a reduction in heart rate and breathing frequency in a sample of 28 preterm infants after hammock positioning was used.

The present study had several limitations. The sampling process was not probabilistic and the sample was small, both of which were factors that limit the extent to which the results can be generalized. A strength of the study was in its novelty and the fact that the findings can be extrapolated to clinical practice.

\section{Conclusions}

Our results showed that hammock positioning was an effective treatment option to reduce pain and improve the sleep-wakefulness state. It also helped to reduce heart rate and breathing frequency, and to increase peripheral $\mathrm{S}_{\mathrm{pO}_{2}}$, which made it another simple, cost-effective tool for treatment of preterm infants in a neonatal ICU.

\section{REFERENCES}

1. Machado Júnior LC, Passini Júnior R, Rodrigues Machado Rosa I. Late prematurity: a systematic review. J Pediatr (Rio J) 2014;90(3):221-231.

2. de Castro MP, Rugolo LM, Margotto PR. [Survival and morbidity of premature babies with less than 32 weeks of gestation in the central region of Brazil.] Rev Bras Ginecol Obstet 2012;34(5):235-242.

3. Santos JA, Procianoy RS, Bohrer BB, Noer C, Librelato GA, Campelo JN. [Do newborn infants feel pain when submitted to gastric suctioning?] J Pediatr (Rio J) 2001;77(5):374-380.

4. Walter-Nicolet E, Annequin D, Biran V, Mitanchez D, Tourniaire B. Pain management in newborn: from prevention to treatment. Paediatr Drugs 2010;12(6):353-365.

5. Franck LS, Greenberg CS, Stevens B. Pain assessment in infants and children. Pediatr Clin North Am 2000;47(3):487-512.

6. Quinn MW, Baker J. Staff perception of pain on a neonatal intensive care unit. Arch Dis Child Fetal Neonatal Ed 2001;85(1):75-78.

7. Paschoalotto G, Eiras PAF, Dellavia PN. Effects of position prone in newborn of neonatal ICU. Reabilitar. 2005;7(27):31-36.

8. Costa KSF, Beleza LO, Souza LM, Ribeiro LM. Hammock position and nesting: comparison of physiological and behavioral effects in preterm infants. Rev Gaúcha Enferm. 2016;37(spe):e62554.

9. Keller A, Arbel N, Merlob P, Davidson S. Neurobehavioral and autonomic effects of hammock positioning in infants with very low birth weight. Pediatr Phys Ther 2003;15(1):3-7.

10. Jesus VR, Oliveira PMN, Azevedo VMGO. Effects of hammock positioning in behavioral status, vital signs, and pain in preterms: a case series study. Braz J Phys Ther 2018;22(4):304-309.

11. Guinsburg R. [Assessing and treating pain in the newborn.] J Pediatr (Rio J) 1999; 75(3):149-160.

12. Silva TP, Silva LJ. Pain scales used in the newborn infant: a systematic review. Acta Med Port 2010;23:437-454.

13. Gavin B, Theodore DW. Handbook of infant development. In: Brazelton TB. Nugent JK. Lester BM, editors. Neonatal behavioral assessment scale. Vol 2. Oxford, England: John Wiley \& Sons; 1987:780-817.

14. Vignochi C, Teixeira PP, Nader SS. Effect of aquatic physical therapy on pain and state of sleep and wakefulness among stable preterm newborns in neonatal intensive care units. Rev Bras Fisiot 2010; 14(3):214-220.

15. Faul F, Erdfelder E, Buchner A, Lang A. Statistical power analyses using $G^{*}$ Power 3.1: tests for correlation and regression analyses. Behav Res Methods. 2009 Nov;41(4):1149-1160.

16. Guinsburg R, Kopelman BI, Almeida MFB, Miyoshi MHI. [Pain in intubated and ventilated preterm neonate: multidimensional assessment and response to fentanyl analgesia.] J Pediat (Rio J) 1994;70(2):82-90.

17. Grunau R. Early pain in preterm infants: a model of long-term effects. Clin Perinatol 2002;29(3):373-394, vii-viii.

18. Corff KE, Seideman R, Venkataraman PS, Lutes L, Yates B. Facilitated tucking: a nonpharmacological comfort measure for pain in preterm neonates. J Obstet Gynecol Neonatal Nurs 1995;24(2):143-147.

19. Geib LT. [Develpment of sleep stages in childhood.] Rev Bras Enferm 2007;60(3):323-326. 
20. Anand KJ. Effects of perinatal pain and stress. Prog Brain Res 2000; 122(1):117-129.

21. Nedivi E. Molecular analysis of developmental plasticity in neocortex. J Neurobiol 1999;41(1):135-147.

22. Orsi KC, Llaguno NS, Avelar AF, Tsunemi MH, Pedreira Mda L, Sato MH, Pinheiro EM. [Effect of reducing sensory and environ- mental stimuli during hospitalized premature infant sleep.] Rev Esc Enferm USP 2015;49(4):550-555.

23. Zanardo V, Trevisanuto D, Dani C, Bottos M, Guglielmi A, Cantarutti F. Oxygen saturation in premature neonates with bronchopulmonary dysplasia in a hammock. Biol Neonate 1995;67(1):54-58.

This article is approved for Continuing Respiratory Care Education credit. For information and to obtain your CRCE

(free to AARC members) visit

www.rcjournal.com 